\title{
Sonographic Diagnosis of Intra-abdominal Pregnancy
}

\author{
${ }^{1}$ Suresh V Phatak, ${ }^{2}$ Neeraj A Patange, ${ }^{3}$ Shishir Rawekar
}

\begin{abstract}
Abdominal pregnancy can be defined as the establishment and development of the fertilized egg partially or entirely in the abdominal cavity, in a primary or more commonly secondary way. This is rarely seen in the evolution of pregnancy, and it accounts for $1 \%$ of the ectopic pregnancy locations. Sonography is important in diagnosis. We are presenting a case report of abdominal pregnancy of 16 weeks gestation.
\end{abstract}

Keywords: Abdominal pregnancy, Extrauterine pregnancy, Sonography.

How to cite this article: Phatak SV, Patange NA, Rawekar S. Sonographic Diagnosis of Intra-abdominal Pregnancy. Int J Recent Surg Med Sci 2016;2(1):52-54.

\section{Source of support: Nil}

Conflict of interest: None

\section{INTRODUCTION}

Peritoneal pregnancies can be classified as primary and secondary. Primary implantation type is extremely rare and is a potentially life-threatening variation of ectopic pregnancy within the peritoneal cavity. Secondary abdominal pregnancies are much more common and result from either tubal abortion or rupture or less often after uterine rupture with subsequent implantation within abdomen. ${ }^{1}$

\section{CASE REPORT}

A woman of 25 years (second gravida) presented with pelvic pain. She earlier had a premature delivery of 7 months and her baby died soon after birth. She was referred for sonography. During examination, a nongravid uterus was seen separate from fetus and placenta (Figs 1 and 2). The fetus was crumpled, and head was deformed (Figs 3A and B). No cardiac activity was noticed. Approximate sonographic gestational age by femur length was 16 weeks. Doppler showed hypervascularity in placenta (Fig. 4). Diagnosis of abdominal pregnancy was given, which was confirmed at surgery (Figs 5 and 6 ).

\footnotetext{
${ }^{1}$ Professor and Head, ${ }^{2,3}$ Resident

${ }^{1-3}$ Department of Radiodiagnosis, Jawaharlal Nehru Medical College, Wardha, Maharashtra, India

Corresponding Author: Suresh V Phatak, Professor and Head, Department of Radiodiagnosis, Jawaharlal Nehru Medical College, Wardha, Maharashtra, India, Phone: +917152287701 e-mail: suresh_phatak@yahoo.com
}

\section{DISCUSSION}

These pregnancies can go undetected until an advanced gestational age and are often complicated by massive hemorrhage. ${ }^{2}$ Important risk factors are tubal damage, pelvic inflammatory disease, endometriosis, assisted reproductive techniques, and multiparity. Clinical history and physical examination alone may not clinch the diagnosis. Sonography is the accurate method for diagnosing an abdominal pregnancy. Magnetic resonance imaging is a complementary imaging modality that helps to confirm the diagnosis and delineate the precise anatomical relationship between the fetus and various maternal abdominal organs. ${ }^{3}$ Salient features for sonographic diagnosis are as follows:

- Demonstration of a fetus in gestational sac outside the uterus or depiction of an abdominal or pelvic mass identifiable as the uterus separate from the fetus.

- Failure to see a uterine wall between the fetus and urinary bladder.

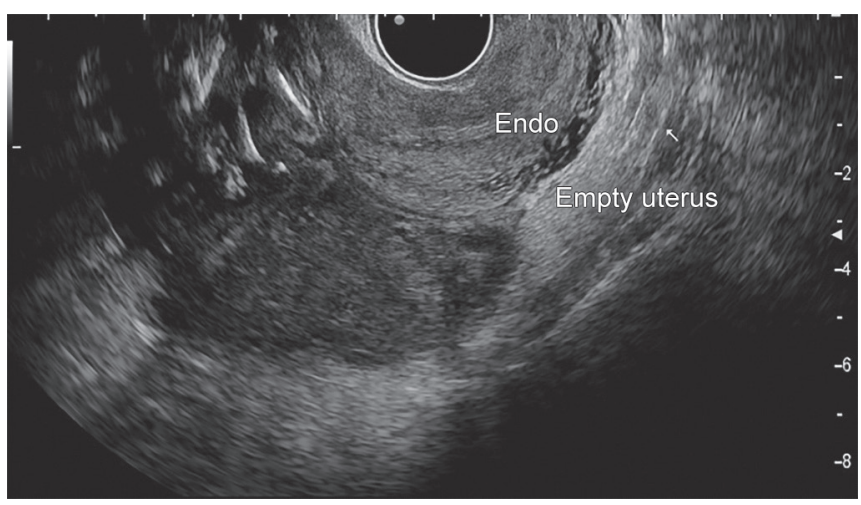

Fig. 1: Ultrasonography shows nongravid uterus and fetal parts (bones) outside the confines of uterus

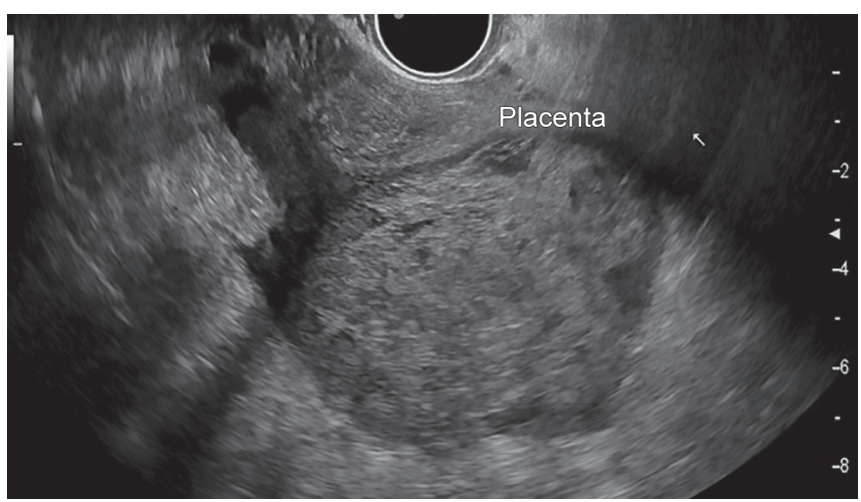

Fig. 2: Ultrasonography shows extrauterine placenta 

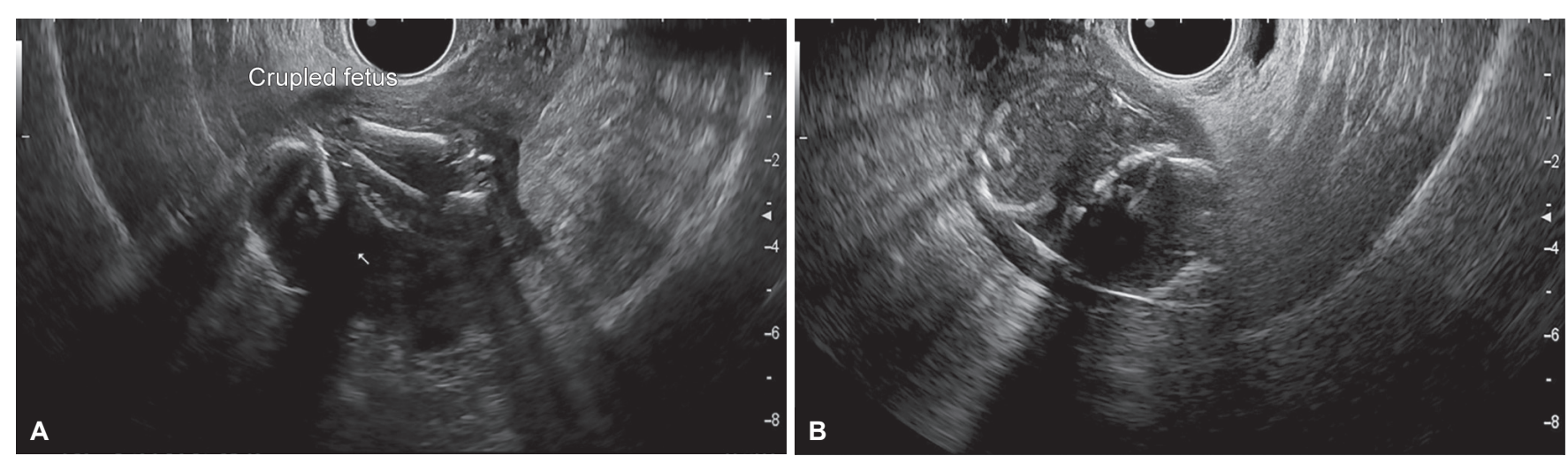

Figs $3 A$ and B: Ultrasonography shows a crumpled fetus

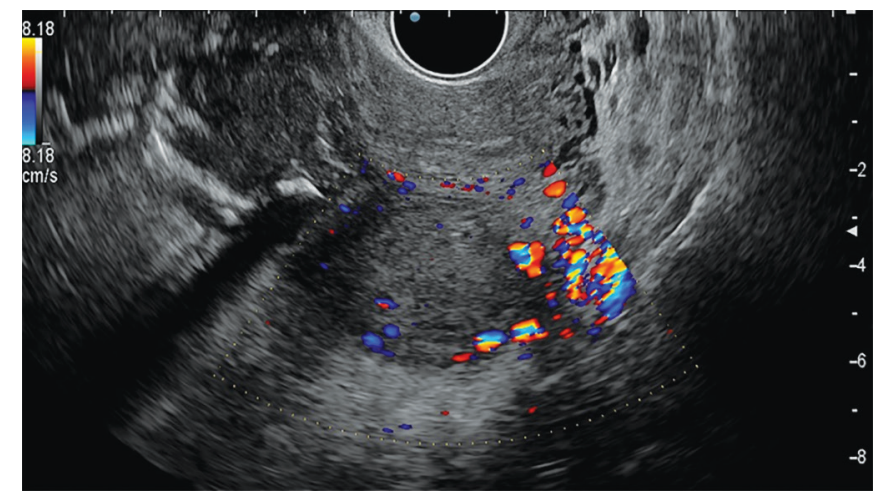

Fig. 4: Color Doppler showing vascularity of placenta

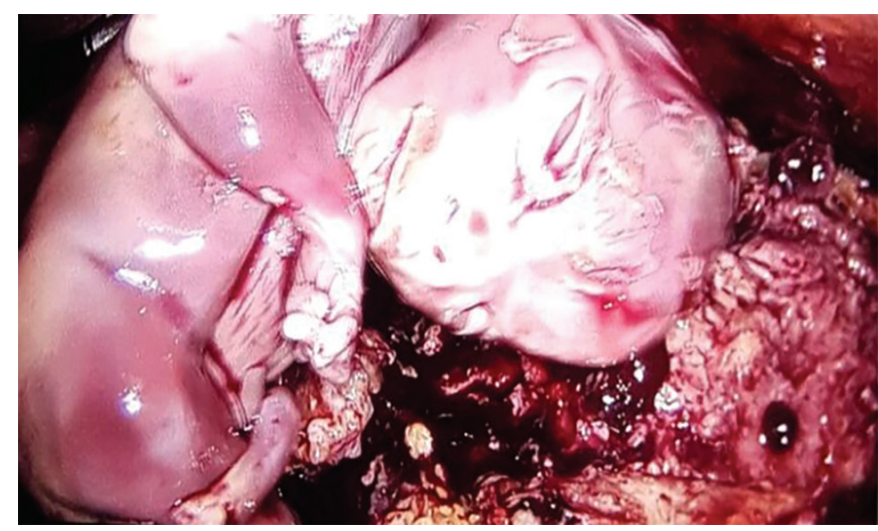

Fig. 6: Intraoperative photographs showing crumpled fetus

- Recognition of a close approximation of fetus to the maternal abdominal wall.

- Localization of placenta outside the confines of uterine cavity. ${ }^{4}$

About $21 \%$ of babies born after an extrauterine abdominal pregnancy have peculiar birth defects, presumably due to compression of the fetus in the absence of amniotic fluid buffer. Typical deformities include limb defects, facial and cranial asymmetry, joint abnormalities, and central nervous malformation. ${ }^{5}$ Ovarian and abdominal ectopic pregnancies are quite rare. On real-time ultrasonography, ovarian ectopic pregnancies move with respect to the ovary, not separately. Abdominal

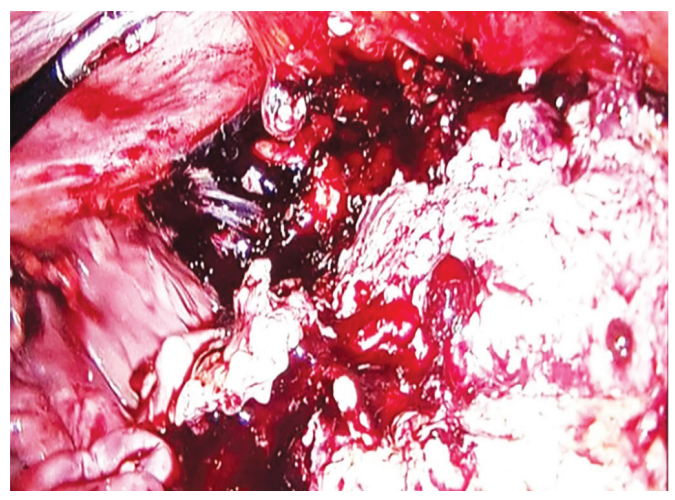

Fig. 5: Intraoperative photographs showing placenta

ectopic pregnancy typically develops in the ligaments of the ovary, and it can get blood supply from the omentum and abdominal organs. At times, these pregnancies migrate out of the pelvis and are located in the upper abdomen. ${ }^{6}$ The chances of survival of a newborn are very poor, with the perinatal mortality rate being 40 to $95 \%$ full term. ${ }^{7}$

\section{CONCLUSION}

Extrauterine abdominal pregnancy is a rare form of ectopic pregnancy. Detailed clinical history, physical examination coupled with dedicated antenatal ultrasound examination is useful to make correct diagnosis. It is associated with high maternal mortality rate. Magnetic resonance imaging is highly specific than other imaging modalities, but due to the cost factor and lack of wide availability, it is less preferred. It can be concluded that careful antenatal ultrasound examination is useful in proper diagnosis of clinically suspected cases of abdominal pregnancies.

\section{REFERENCES}

1. Shin JS, Moon YJ, Kim SR, Kim KT, Moon H, Hwang YY. Primary peritoneal pregnancy implanted on uterosacral ligament: a case report. J Korean Med Sci 2000 Jun;15(3): 359-362. 
2. Kun KY, Wong PY, Ho MW, Tai CM, Ng TK. Abdominal pregnancy presenting as missed abortion at 16 weeks' gestation. Hong Kong Med J 2000 Dec;6(4):425-427.

3. Radhakrishnan K. Intra-abdominal pregnancy. Appl Radiol 2015 May;44(5):44-47.

4. Allibone GW, Fagan CJ, Porter SC. The sonographic features of intra-abdominal pregnancy. J Clin Ultrasound 1981 Sep;9(7):383-387.
5. Stevens CA. Malformations and deformations in abdominal pregnancy. Am J Med Genet 1993 Dec;47(8):11891195.

6. Levine D. Ectopic pregnancy. Radiology 2007 Nov;245(2): 385-396.

7. Martin JN Jr, Sessums JK, Martin RW, Pryor JA, Morrison JC. Abdominal pregnancy: current concepts of management. Obstet Gynecol 1988 Apr;71(4):549-557. 